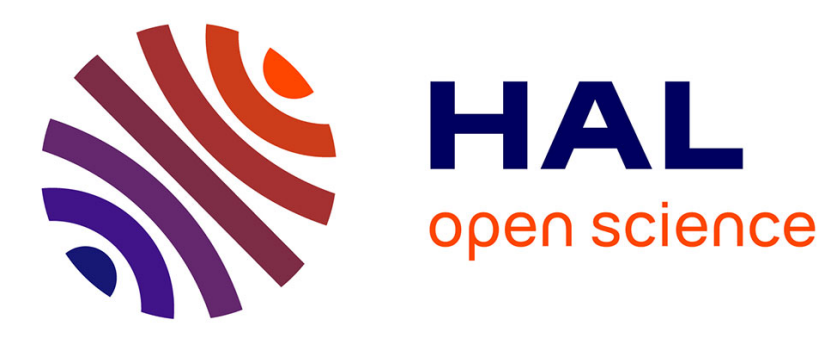

\title{
Möbius inversion formula for the trace group
}

\author{
Anne Bouillard, Jean Mairesse
}

\section{To cite this version:}

Anne Bouillard, Jean Mairesse. Möbius inversion formula for the trace group. Comptes rendus de l'Académie des sciences. Série I, Mathématique, 2003, 339 (12), pp.899-904. 10.1016/j.crma.2004.10.017 . inria-00099682

\section{HAL Id: inria-00099682 \\ https://hal.inria.fr/inria-00099682}

Submitted on 27 Jul 2007

HAL is a multi-disciplinary open access archive for the deposit and dissemination of scientific research documents, whether they are published or not. The documents may come from teaching and research institutions in France or abroad, or from public or private research centers.
L'archive ouverte pluridisciplinaire HAL, est destinée au dépôt et à la diffusion de documents scientifiques de niveau recherche, publiés ou non, émanant des établissements d'enseignement et de recherche français ou étrangers, des laboratoires publics ou privés. 


\title{
Möbius inversion formula for the trace group
}

\author{
Anne Bouillard a ${ }^{\text {and Jean Mairesse }}{ }^{\text {a }}$ \\ a LiAfa, CNRS - Université Paris 7 - Case 7014 - 2, place Jussieu - 75251 Paris Cedex 05 - France. \\ E-mail:\{bouillard, mairesse $\} @$ liafa.jussieu.fr
}

(Reçu le jour mois année, accepté après révision le jour mois année)

\begin{abstract}
A trace group (monoid) is the quotient of a free group (monoid) by relations of commutation between some pairs of generators. We prove an analog for the trace group of the Möbius inversion formula for the trace monoid (Cartier and Foata, 1969). (C) 200? Académie des sciences/Éditions scientifiques et médicales Elsevier SAS
\end{abstract}

\section{Une formule d'inversion de Möbius pour le groupe de traces}

Résumé. Un groupe (monoïde) de traces est le quotient d'un groupe (monoïde) libre par des relations de commutation entre certaines paires de générateurs. On montre un analogue pour le groupe de traces de la formule d'inversion de Möbius pour le monoüde de trace (Cartier et Foata, 1969). (C) 200? Académie des sciences/Éditions scientifiques et médicales Elsevier SAS

\section{Version française abrégée}

Un groupe (monoïde) de traces est le quotient d'un groupe (monoïde) libre par des relations de commutation entre certaines paires de générateurs (lettres). Les monoïdes de traces sont classiquement utilisés pour modéliser les exécutions dans les systèmes concurrents, voir [5] et les références qui s'y trouvent. Entre autres intérêts, les groupes de traces permettent d'“approximer" les groupes de tresses [16].

Un élément $m$ d'un monoïde de traces $M$ est une clique si chaque lettre le composant apparait au plus une fois, et si toutes ces lettres commutent. On note $\mathfrak{C}$ l'ensemble des cliques de $M$. Soit $|m|$ la longueur de $m \in M$ définie en (3). Soit $\mu_{M} \in \mathbb{Z}\langle M\rangle$ le polynôme défini par $\mu_{M}=\sum_{c \in \mathfrak{C}}(-1)^{|c|} c$. Dans [3, Théorème 2.4], il est prouvé que $\mu_{M}$ est l'inverse formel dans $\mathbb{Z}\langle\langle M\rangle\rangle$ de la série caractéristique de $M$, c'est-à-dire que l'on a l'identité (4). On appelle cette identité la 'formule d'inversion de Möbius pour le monoïde de traces'. Elle est au cœur de l'étude combinatoire du monoïde de traces [4, 5].

On obtient dans cette note une 'formule d'inversion de Möbius pour le groupe de traces'. Soit $F$ un groupe de traces sur l'alphabet $\Sigma \cup \bar{\Sigma}(\bar{a} \in \bar{\Sigma}$ est l'inverse de $a \in \Sigma)$. Soit $M$ le monoïde de traces sur $\Sigma \cup \bar{\Sigma}$ dont la présentation de monoïde est obtenue à partir de la présentation de monoïde de $F$ en supprimant les relations $a \bar{a}=\bar{a} a=1, a \in \Sigma$. Soit $\phi$ l'injection canonique de $F$ dans $M$. (Plus précisément, $\phi(t), t \in F$, est la projection dans $M$ d'un représentant de longueur minimale de $t$ dans $(\Sigma \cup \bar{\Sigma})^{*}$.) Soit $\mathfrak{D}=T(\mathfrak{C})$ où

\section{Note présentée par M. Nivat}

S0764-4442(00)0????-?/FLA

(c) 200? Académie des sciences/Éditions scientifiques et médicales Elsevier SAS. Tous droits réservés. 


\section{A. Bouillard and J. Mairesse}

$T: M \rightarrow \mathcal{P}(M)$ est la fonction définie $\operatorname{par} T(a)=a(\bar{a} a)^{*}(1+\bar{a}), a \in \Sigma \cup \bar{\Sigma}$, et étendue par morphisme. On démontre que $\left(\sum_{d \in \mathfrak{D}}(-1)^{|d|} d\right)$ est l'inverse formel dans $\mathbb{Z}\langle\langle M\rangle\rangle$ de la série caractéristique de $\phi(F)$, c'est-à-dire que l'on a l'identité (7). Une conséquence de cette identité est que la série de croissance de $F$ s'exprime comme : $\sum_{t \in F} x^{|t|}=\sum_{t \in M} x^{|t|} /(1+x)^{|t|}=\left(\sum_{c \in \mathfrak{C}}(-1)^{|c|} x^{|c|} /(1+x)^{|c|}\right)^{-1}$. Ceci permet d'exporter vers le groupe de traces les résultats prouvés dans $[10,12]$ pour le monoïde de traces.

\section{Introduction}

A decomposition of an element $m$ of a monoid $M$ is a $n$-uple $\left(m_{1}, \ldots, m_{n}\right)$ satisfying $m_{1} \cdots m_{n}=m$ with $m_{i} \in M \backslash\{1\}$ (where 1 is the unit of the monoid). The decomposition is even (odd) if $n$ is even (odd). Assume any element of $M$ has a finite number of decompositions and set $\left(\mu_{M} \mid m\right)$ to be the number of even decompositions of $m$ minus the number of odd decompositions. View $\mu_{M}$ as a formal series of $\mathbb{Z}\langle\langle M\rangle\rangle$ and call it the Möbius series of $M$. A one line computation shows that $\mu_{M}$ is the formal inverse of the characteristic series of $M$ : in $\mathbb{Z}\langle\langle M\rangle\rangle,\left(\sum_{m \in M} m\right) \cdot \mu_{M}=1$. This identity is called a Möbius inversion formula, see Cartier and Foata [3], Lallement [13], or Rota [15] in a different setting. The classical Möbius inversion principle in number theory (see [11], Chapter XVI) is a special instance of the identity. It is generally difficult to effectively compute the Möbius series.

A trace group (monoid) is the quotient of a free group (monoid) by relations of commutation between some pairs of generators (letters). Observe that in a trace monoid, the traces all have a finite number of decomposions. A classical result of Cartier and Foata [3, Theorem 2.4] states that the Möbius series of a trace monoid is a polynomial which is explicitly computable, see $\S 3$. This is the starting point of the combinatorial study of the trace monoid $[4,5]$. In a trace group as in any non-trivial group, the elements do not have a finite number of decompositions and the Möbius series is not defined. However, we prove in this note an identity which has the flavor of a Möbius inversion formula, see Theorem 4.1. As a by-product we express the growth series of a trace group in fonction of the growth series of a canonically associated trace monoid, see Corollary 4.2.

Trace monoids are often used to model the occurrence of events in concurrent systems, see [5] and the references therein. Roughly speaking, a letter corresponds to an event and two letters commute when the corresponding events can occur simultaneously. Trace groups have been studied from several viewpoints (and under various names like graph groups or right angled Artin groups) [6, 8, 16]. An important motivation is that trace groups can 'approximate' braid groups [16].

\section{Trace monoids and trace groups}

Let $\Sigma$ be a non-empty finite set and denote by $\Sigma^{*}$ the free monoid over $\Sigma$. The empty word is denoted by 1. The monoid presented by $\Sigma$ and $R \subset \Sigma^{*} \times \Sigma^{*}$ is denoted by $\langle\Sigma \mid u=v,(u, v) \in R\rangle$. Let $I \subset \Sigma \times \Sigma$ be an anti-reflexive and symmetric relation, called an independence (or commutation) relation. The trace monoid (or free partially commutative monoid) $\mathbb{M}(\Sigma, I)$ is defined by the monoid presentation

$$
\mathbb{M}(\Sigma, I)=\langle\Sigma \mid a b=b a, \forall(a, b) \in I\rangle
$$

The elements of $\mathbb{M}(\Sigma, I)$ are called traces.

Let $\bar{\Sigma}$ be a copy of $\Sigma$, that is a set which is in bijection with $\Sigma$ and disjoint from $\Sigma$; to each letter $a \in \Sigma$ corresponds the letter $\bar{a}$ in $\bar{\Sigma}$. Set $\widetilde{\Sigma}=\Sigma \cup \bar{\Sigma}$. We extend the bar notation to $\widetilde{\Sigma}$, by setting $\overline{\bar{a}}=a$. Denote by $\mathbb{F}(\Sigma)$ the free group over $\Sigma$, with $\bar{\Sigma}$ as the set of inverses of the generators. Let $\mathbb{F}(\Sigma, I)$ be defined by the monoid presentation

$$
\mathbb{F}(\Sigma, I)=\langle\widetilde{\Sigma} \mid a \bar{a}=\bar{a} a=1, \forall a \in \Sigma ; a b=b a, \forall(a, b) \in I\rangle .
$$


It is easily checked that $\mathbb{F}(\Sigma, I)$ is a group, called the trace group (or free partially commutative group). A group presentation of the trace group is $\mathbb{F}(\Sigma, I)=\langle\Sigma \mid a b=b a, \forall(a, b) \in I\rangle$. The elements of $\mathbb{F}(\Sigma, I)$ are also called traces.

When $I=\varnothing$, the corresponding trace monoid is the free monoid $\Sigma^{*}$ and the corresponding trace group is the free group $\mathbb{F}(\Sigma)$. When $I=\Sigma \times \Sigma \backslash\{(a, a), a \in \Sigma\}$, the corresponding trace monoid is the free commutative monoid $\mathbb{N}^{\Sigma}$ and the corresponding trace group is the free commutative group $\mathbb{Z}^{\Sigma}$.

Define $\iota: \widetilde{\Sigma} \longrightarrow \Sigma$ by: $\forall a \in \Sigma, \iota(a)=\iota(\bar{a})=a$. The relation $\widetilde{I} \subset \widetilde{\Sigma} \times \widetilde{\Sigma}$ is defined by

$$
(u, v) \in \widetilde{I} \Longleftrightarrow(\iota(u), \iota(v)) \in I
$$

The diagram below, where the applications are the canonical surjective morphisms, is commutative.

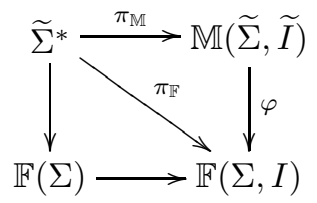

The notations are shortened to $\pi$ and $\varphi$ when there is no possible confusion. It is convenient to denote a trace by any of its word representatives, that is to identify $w$ and $\pi(w)$. For instance, 1 denotes the empty trace. Given a word $w \in \Sigma^{*}$ and a letter $a \in \Sigma$, denote by $|w|_{a} \in \mathbb{N}$ the number of occurrences of the letter in the word. We now give several definitions which are stated in order to hold both in a trace monoid and in a trace group. For the trace monoid only, the same definitions take a simpler form. Let $S$ be the set of generators (including the inverses for the group). The length (with respect to $S$ ) of a trace $t$ is:

$$
|t|=\min \left\{k \mid t=g_{1} \cdots g_{k}, g_{i} \in S\right\} .
$$

Observe that $|t|=\min \left\{|x|, x \in \pi^{-1}(t)\right\}$. Given a trace $t$ and a letter $a$, define $|t|_{a}=\min \left\{|x|_{a}, x \in\right.$ $\left.\pi^{-1}(t)\right\}$, and define the alphabet of $t$ by $\operatorname{alph}(t)=\left\{\left.a \in S|| t\right|_{a}>0\right\}$. A trace $t$ is a clique if: $\forall a \in$ $\operatorname{alph}(t),|t|_{a}=1$ and $\forall a, b \in \operatorname{alph}(t), a b=b a$. We denote the set of cliques by $\mathfrak{C}$. Observe that $1 \in \mathfrak{C}$.

We visualize traces using Viennot's representation as heaps of pieces [17, 16]. In the trace monoid, a trace corresponds to a heap. In the trace group, a trace corresponds to a colored heap (letters of $\Sigma$ are associated with light gray pieces, letters of $\bar{\Sigma}$ with dark gray pieces, and, in the colored heap, consecutive pieces of type $a$ and $\bar{a}$ cancel each other).

Example 1. - The Basic Example to be used as illustration throughout the paper consists of $\Sigma=\{a, b, c\}$ and $I=\{(a, b),(b, a)\}$. We have represented in Figure 1 , the traces in $\mathbb{M}(\widetilde{\Sigma}, \widetilde{I})$ and $\mathbb{F}(\Sigma, I)$ corresponding
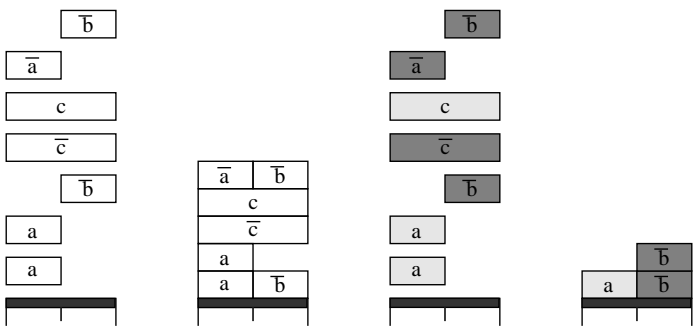

Figure 1: A heap in $\mathbb{M}(\widetilde{\Sigma}, \widetilde{I})$ (left) and a colored heap in $\mathbb{F}(\Sigma, I)$ (right).

to the word $u=a a \bar{b} \bar{c} c \bar{a} \bar{b}$. We have $\left|\pi_{\mathbb{M}}(u)\right|=7$ and $\left|\pi_{\mathbb{F}}(u)\right|=3$. 


\section{A. Bouillard and J. Mairesse}

The heap representation enables to define the Cartier-Foata normal form for traces by reading a trace 'slice by slice' in the associated heap [3]. A corollary is that the word problem is solvable in polynomial time and that trace groups (monoids) are automatic in the sense of [9]. Another consequence is the following, see [7, Prop. 2.4.7] for a formal proof. Given $u, v \in \widetilde{\Sigma}^{*}$ such that $\pi_{\mathbb{F}}(u)=\pi_{\mathbb{F}}(v)$ and $|u|=|v|=\left|\pi_{\mathbb{F}}(u)\right|$, then we have $\pi_{\mathbb{M}}(u)=\pi_{\mathbb{M}}(v)$. In other words, a trace of $\mathbb{F}(\Sigma, I)$ admits a unique representative of minimal length in $\mathbb{M}(\widetilde{\Sigma}, \widetilde{I})$. We denote by $\phi: \mathbb{F}(\Sigma, I) \longrightarrow \mathbb{M}(\widetilde{\Sigma}, \widetilde{I})$ the corresponding map. Using the language of heaps, if $t$ is a colored heap then $\phi(t)$ is the corresponding (non-colored) heap obtained by forgetting the colors.

Let $M$ be a trace monoid or a trace group. The growth series of $M$ is the series $\operatorname{Gr} M \in \mathbb{Z} \llbracket x \rrbracket$ defined by $\operatorname{Gr} M=\sum_{m \in M} x^{|m|}=\sum_{n \in \mathbb{N}} \#\{m \in M|| m \mid=n\} x^{n}$. Set $a_{n}=\#\{m \in M|| m \mid=n\}$. The growth rate of $M$ is $\rho_{M}=\lim _{n} a_{n}^{1 / n} \geqslant 1$ (the limit exists by sub-additivity of the sequence $\left(\log a_{n}\right)_{n}$ ).

\section{Möbius formula for the trace monoid}

The results recalled here are classical and due to Cartier and Foata [3, Theorem 2.4]. Let $\mathbb{M}(\Sigma, I)$ be a trace monoid. Denote by $\mathbb{Z}\langle\langle\mathbb{M}(\Sigma, I)\rangle\rangle$ the ring of formal power series over $\mathbb{M}(\Sigma, I)$ with coefficients in $\mathbb{Z}$. This ring is cancellative, in particular a right or left inverse is an inverse. When $\mathbb{M}(\Sigma, I)=\{x\}^{*}$, we write $\mathbb{Z} \llbracket x \rrbracket$ for $\mathbb{Z}\langle\langle\mathbb{M}(\Sigma, I)\rangle\rangle$. The following identity holds in $\mathbb{Z}\langle\langle\mathbb{M}(\Sigma, I)\rangle$ :

$$
\left(\sum_{t \in \mathbb{M}(\Sigma, I)} t\right) \cdot\left(\sum_{c \in \mathfrak{C}}(-1)^{|c|} c\right)=1 .
$$

Using the teminology of the Introduction, the Möbius series of $\mathbb{M}(\Sigma, I)$ is $\mu_{\mathbb{M}(\Sigma, I)}=\sum_{c \in \mathfrak{C}}(-1)^{|c|} c$. Define $\mu_{\mathbb{M}(\Sigma, I)}(x) \in \mathbb{Z} \llbracket x \rrbracket$ by $\mu_{\mathbb{M}(\Sigma, I)}(x)=\sum_{c \in \mathfrak{C}}(-1)^{|c|} x^{|c|}$. An easy consequence of (4) is the following identity in $\mathbb{Z} \llbracket x \rrbracket$ :

$$
\operatorname{GrM}(\Sigma, I) \cdot \mu_{\mathbb{M}(\Sigma, I)}(x)=1 .
$$

So, the growth series of the trace monoid is the formal inverse of $\mu_{\mathbb{M}(\Sigma, I)}(x)$. The objective is to get analogs of (4) and (5) for trace groups.

\section{Möbius formula for the trace group}

Define the set of alternate traces as the subset $\mathfrak{D}$ of $\mathbb{M}(\widetilde{\Sigma}, \widetilde{I})$ given by the regular expression

$$
\mathfrak{D}=\sum_{c \in \mathfrak{C}}\left[\prod_{x \in \operatorname{alph}(c)} x(\bar{x} x)^{*}(1+\bar{x})\right],
$$

where $\mathfrak{C}$ is the set of cliques of $\mathbb{M}(\widetilde{\Sigma}, \widetilde{I})$. Figure 2 shows some alternate traces for the Basic Example.
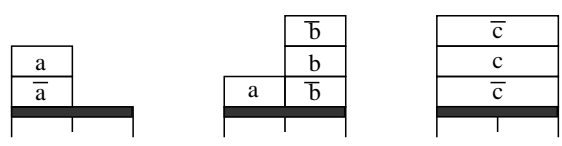

Figure 2: Examples of elements of $\mathfrak{D}$.

THEOREM 4.1. - In $\mathbb{Z}\langle\langle\mathbb{M}(\widetilde{\Sigma}, \widetilde{I})\rangle$, we have the following identity:

$$
\left(\sum_{t \in \phi(\mathbb{F}(\Sigma, I))} t\right) \cdot\left(\sum_{d \in \mathfrak{D}}(-1)^{|d|} d\right)=1 .
$$


Proof. - We are going to use the same type of bijective argument as in [17, Remark 5.2]. For a trace $t \in \mathbb{M}(\widetilde{\Sigma}, \widetilde{I})$, define $\operatorname{Top}(t)=\{a \in \operatorname{alph}(t) \mid \exists u, t=u a\}$. The set $\operatorname{Top}(t)$ contains all the pieces which are fully visible when the heap $t$ is viewed from above. Given $t \in \mathbb{M}(\widetilde{\Sigma}, \widetilde{I})$ and $h \in \mathfrak{D}$, define $M(t, h) \subset \Sigma$ by $M(t, h)=\iota(\operatorname{Top}(h) \cup\{a \in \operatorname{Top}(t) \mid \forall b \in \operatorname{Top}(h), a \widetilde{I} b\})$. Observe that $M(t, h)=\varnothing$ if and only if $(t, h)=(1,1)$. We now define an application

$$
\psi: \phi(\mathbb{F}(\Sigma, I)) \times \mathfrak{D} \longrightarrow \phi(\mathbb{F}(\Sigma, I)) \times \mathfrak{D}
$$

We have $\psi(1,1)=(1,1)$. For $(t, h) \in \phi(\mathbb{F}(\Sigma, I)) \times \mathfrak{D}$ with $(t, h) \neq(1,1)$, we distinguish between four cases. Equip $\Sigma$ with a total order and let $a$ be the smallest letter of $M(t, h)$.

1. If we have $t=t_{1} a_{1}$ with $a_{1} \in\{a, \bar{a}\}$ and $a, \bar{a} \notin \operatorname{alph}(h)$, then $\psi(t, h)=\left(t_{1}, a_{1} h\right)$.

2. If we have $h=a_{1} h_{1}$ with $a_{1} \in\{a, \bar{a}\}$ and $a, \bar{a} \notin \operatorname{Top}(t)$, then $\psi(t, h)=\left(t a_{1}, h_{1}\right)$.

3. If we have $t=t_{1} a_{1}$ with $a_{1} \in\{a, \bar{a}\}$ and $h=a_{1} h_{1}$, then $\psi(t, h)=\left(t a_{1}, h_{1}\right)$.

4. If we have $t=t_{1} a_{1}$ with $a_{1} \in\{a, \bar{a}\}$ and $h=\bar{a}_{1} h_{1}$, then $\psi(t, h)=\left(t_{1}, a_{1} h\right)$.

The dynamics of $\psi$ for the Basic Example is illustrated in Figure 3. Here the order is $a<b<c$.

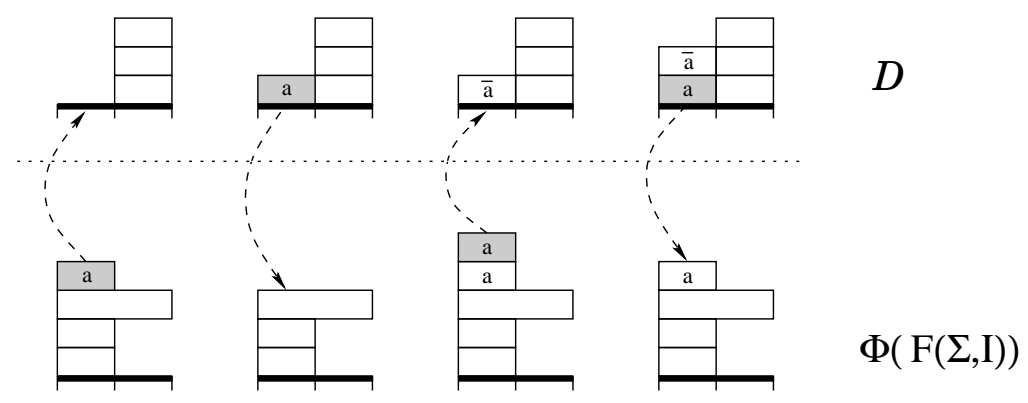

Figure 3: The involution $\psi$. From left to right, the cases are 1,2,4, and 3.

Observe that the configurations of type $a a$ are allowed in $\phi(\mathbb{F}(\Sigma, I))$ but not in $\mathfrak{D}$, and the other way round the configurations of type $a \bar{a}$ are allowed in $\mathfrak{D}$ but not in $\phi(\mathbb{F}(\Sigma, I))$. Notice also that the smallest letters of $M(t, h)$ and $M \psi(t, h)$ are the same. It follows easily from these two points that $\psi^{2}=$ Id. It is also clear that the only fixed point of $\psi$ is $(1,1)$. Furthermore, if $(t, h) \neq(1,1)$ and $\psi(t, h)=\left(t^{\prime}, h^{\prime}\right)$, then we have $t h=t^{\prime} h^{\prime}$ and $\left|h^{\prime}\right|=|h| \pm 1$. So, we have

$$
(-1)^{|h|} t h=-(-1)^{\left|h^{\prime}\right|} t^{\prime} h^{\prime}
$$

Set $S=\phi(\mathbb{F}(\Sigma, I)) \times \mathfrak{D}$. By decomposing the sum over the orbits of $\psi$, we get

$$
\left(\sum_{t \in \phi(\mathbb{F}(\Sigma, I))} t\right) \cdot\left(\sum_{h \in \mathfrak{D}}(-1)^{|h|} h\right)=1+\sum_{(t, h) \in S \backslash\{(1,1)\}}(-1)^{|h|} t h=1 .
$$




\section{A. Bouillard and J. Mairesse}

COROLlARY 4.2. - In $\mathbb{Z} \llbracket x \rrbracket$, the following identity holds:

$$
\operatorname{Gr} \mathbb{F}(\Sigma, I) \cdot \mu_{\mathbb{M}(\widetilde{\Sigma}, \widetilde{I})}(x /(1+x))=1 .
$$

Corollary 4.2 appears in Lewin [14], with a quite different proof from which the stronger statement in Theorem 4.1 cannot be deduced. So the growth series is a rational series with a simple combinatorial definition. Observe that $\mu_{\mathbb{M}(\widetilde{\Sigma}, \widetilde{I})}(x)=\mu_{\mathbb{M}(\Sigma, I)}(2 x)$. Since the growth rate is the inverse of the modulus of a dominant singularity, we obtain: $\rho_{\mathbb{F}(\Sigma, I)}=\rho_{\mathbb{M}(\widetilde{\Sigma}, \widetilde{I})}-1=2 \rho_{\mathbb{M}(\Sigma, I)}-1$. A consequence is that the results originally proved for $\rho_{\mathbb{M}(\Sigma, I)}$ in $[10,12]$ can be directly transferred to $\rho_{\mathbb{F}(\Sigma, I)}$. For instance, $\operatorname{Gr} \mathbb{F}(\Sigma, I)$ has a unique dominant singularity which is positive real. If $(\Sigma,(\Sigma \times \Sigma) \backslash I)$ is a connected graph, then this dominant singularity is of order 1 and consequently $\#\{t \in \mathbb{F}(\Sigma, I),|t|=n\} \sim c \rho_{\mathbb{F}(\Sigma, I)}^{n}$.

We conclude by listing some complementary results, for details see $[1,2]$. The identity $(7)$ can be lifted to the free monoid under some conditions on $(\Sigma, I)$ (this is a consequence of [4, Theorem 4.4.2]). The height $h(t)$ of a trace $t$ is the height of the corresponding heap. More formally, the height is the length defined in (3) with respect to the set of generators $\mathfrak{C} \backslash\{1\}$. The average height $\lambda=\lim _{n} n^{-1}\left(\sum_{|t|=n} h(t)\right) /\left(\sum_{|t|=n} 1\right)$ is an explicitly computable algebraic number. The proof uses Corollary 4.2 in a preeminent way.

Example 2. - Consider the Basic Example. Applying Theorem 4.1, the characteristic series of $\phi(\mathbb{F}(\Sigma, I))$ is the formal inverse of $1-\sum_{x \in \widetilde{\Sigma}} x(\bar{x} x)^{*}(\bar{x}-1)+\sum_{\iota(x)=a, \iota(y)=b} x(\bar{x} x)^{*}(\bar{x}-1) y(\bar{y} y)^{*}(\bar{y}-1)$. Using (5), we have $\operatorname{GrM}(\widetilde{\Sigma}, \widetilde{I})=\left(1-6 x+4 x^{2}\right)^{-1}$. Applying Corollary 4.2, we get the growth series $\operatorname{Gr} \mathbb{F}(\Sigma, I)=(1+x)^{2} /\left(1-4 x-x^{2}\right)$. The growth rate of the trace group is $(\sqrt{5}+2)$. The average height is $\lambda=(25-3 \sqrt{5}) / 20$.

\section{References}

[1] A. Bouillard. Rapport de DEA : Le groupe de traces. LIAFA research report 2002-13, Université Paris 7, 2002.

[2] A. Bouillard and J. Mairesse. Generating series of the trace group. In Z. Ésik and Z. Fülöp, editors, Developments in Language Theory, volume 2710 of LNCS, pages 159-170. Springer-Verlag, 2003.

[3] P. Cartier and D. Foata. Problèmes combinatoires de commutation et réarrangements. Number 85 in Lecture Notes in Mathematics. Springer, 1969.

[4] V. Diekert. Combinatorics on traces. Number 454 in LNCS. Springer Verlag, 1990.

[5] V. Diekert and G. Rozenberg, editors. The Book of Traces. World Scientific, 1995.

[6] C. Droms, B. Servatius, and H. Servatius. Groups assembled from free and direct products. Discrete Math., 109:69-75, 1992

[7] C. Duboc. Commutations dans les monoïdes libres : un cadre théorique pour l'étude du parallélisme. $\mathrm{PhD}$ thesis, Université de Rouen, 1986. Also LITP Report 86-25, Univ. Paris 7.

[8] G. Duchamp and D. Krob. Partially commutative Magnus transformations. Int. J. Alg. Comput., 3:15-41, 1993.

[9] D. Epstein, J. Cannon, D. Holt, S. Levy, M. Paterson, and W. Thurston. Word processing in groups. Jones and Bartlett, Boston, 1992.

[10] M. Goldwurm and M. Santini. Clique polynomials have a unique root of smallest modulus. Information Processing Letters, 75(3):127-132, 2000.

[11] G. Hardy and E. Wright. An introduction to the theory of numbers. Clarendon Press, Oxford, 1979. 5-th edition.

[12] D. Krob, J. Mairesse, and I. Michos. Computing the average parallelism in trace monoids. Discrete Math., 273:131-162, 2003.

[13] G. Lallement. Semigroups and Combinatorial Applications. Wiley, New-York, 1979.

[14] J. Lewin. The growth function of a graph group. Comm. Algebra, 17(5):1187-1191, 1989.

[15] G.-C. Rota. On the foundations of combinatorial theory. I. Theory of Möbius functions. Z. Wahrscheinlichkeitstheor. Verw. Geb., 2:340-368, 1964.

[16] A. Vershik, S. Nechaev, and R. Bikbov. Statistical properties of locally free groups with applications to braid groups and growth of random heaps. Commun. Math. Phys., 212(2):469-501, 2000.

[17] G.X. Viennot. Heaps of pieces, I: Basic definitions and combinatorial lemmas. In Labelle and Leroux, editors, Combinatoire Énumérative, number 1234 in Lect. Notes in Math., pages 321-350. Springer, 1986. 